\title{
OCORRÊNCIA DE VETORES BIOLÓGICOS DA Dermatobia hominis (L.Jr., 1781) (Díptera: Cuterebridae), CAPTURADOS COM ARMADILHA MAGOOM NA REGIÃO METALÚRGICA DO ESTADO DE MINAS GERAIS, BRASIL
}

\author{
OCURRENCE OF BIOLOGICAL VECTORS OF Dermatobia hominis (L. Jr.,1781)(Diptera:Cuterebridae), \\ CAPTURED BY MAGOOM TRAP IN THE MATALLURGIC REGION, MINAS GERAIS, BRAZIL.
}

\author{
Batista Zenón Rodríguez ${ }^{1}$ Romário Cerqueira Leite ${ }^{2}$
}

\section{RESUMO}

\begin{abstract}
Através do uso da armadilha Magoom foram capturados 49.010 insetos no periodo de junho de 1995 a maio de 1996 no municipio de Pedro Leopoldo MG. Foram identificadas 10 ordens de insetos. Destes, $95,73 \%$ pertencem à ordem Diptera. Três familias, Sepsidae, Syrphidae e Culicidae participaram com $24,65 \%, 18,53 \%$, e $17,64 \%$ respectivamente dos 46.915 dipteros capturados. Dos três locais pesquisados, a mata (local 3) contribuiu com o maior número de insetos capturados correspondentes a 43,99\% do total de espécimes colhidos. Os vetores biológicos de D. hominis (com postura aderida) importantes por ordem de frequência foram Fannia spp, Musca domestica, Stomoxys calcitrans, Synthesiomyia nudiseta, Ophira sp., sendo esta última notificada pela primeira vez. O gênero Fannia spp. apresentou-se como de maior importância epidemiológica por sua maior freqüência e distribuição nos locais de captura.
\end{abstract}

Palavras-chave: Dermatobia hominis, vetores biológicos, dermatobiose, diptera.

\section{SUMMARY}

From June 1995 to May 1996, 49,010 insects were captured in the municipality of Pedro Leopoldo, MG, Brazil, using the Magoom's trap. Ten orders were identified and $95.73 \%$ of them belong to the order Diptera. Three families, Sepsidae, Syrphidae, and Culicidae, represented $24.65 \%, 18.53 \%$, and $17.64 \%$ respectively, of 46,915 diptera trapped. The bush contributed with the highest number of trapped insects $(43.99 \%)$ when compared with stable or pasture. The biological vectors of D. hominis (with adhered, eggs), in order of prevalence, were Fannia spp, Musca domestica, Stomoxys calcitrans, Synthesiomyia nudiseta, Ophira sp..This is the first identification of the genus Ophira sp. as biological vector of $D$. hominis. The genus Fannia was found to be the most important biological vector of $D$. hominis in Pedro Leopoldo, MG, Brazil, because of its high frequency and distribution in the three environment conditions searched.

Key words: Dermatobia hominis, biological vectors, dermatobiose, diptera.

\section{INTRODUÇÃO}

A Dermatobia hominis diferencia-se radicalmente na sua biologia de outros muscoides, pelo fato de depender de outros dípteros como vetores biológicos para dar continuidade a seu ciclo biológico. Este evento foi observado pela primeira vez por Morales, na Guatemala, em 1911 de acordo com NEIVA \& GOMES (1917). No passado o carrapato Amblyomma cajennense também foi considerado como possível vetor biológico da $\boldsymbol{D}$. hominis (DUNN, 1918), mas esta afirmação foi contestada por NEEL $\boldsymbol{e t}$ al. (1955).

\footnotetext{
${ }^{1}$ Médico Veterinário e Zootecnista, aluno do Curso de Pós-graduação em Parasitologia, Departamento de Parasitologia, Instituto de Ciências Biológicas, Universidade Federal de Minas Gerais (UFMG).

${ }^{2}$ Médico Veterinário, Professor Adjunto, Departamento de Medicina Veterinária Preventiva, Escola de Veterinária, UFMG, Campus da Pampulha, 31270-010, Belo Horizonte, MG, Brasil. Autor para correspondência 
ARTIGAS \& SERRA (1965) atualizaram uma lista de vetores biológicos de D. hominis, anteriormente descrita por NEEL et al. (1955), a qual citava muitas espécies de dípteros das famílias Simulidae, Muscidae, Calliphoridae, e Tabanidae, a seguir a de GUIMARÃES et al. (1983) que assinalaram inúmeras espécies de dípteros, como vetores biológicos da $\boldsymbol{D}$. hominis, pertencentes às famílias Culicidae, Simulidae, Tabanidae, Fanniidae, Anthomyidae, Muscidae, Sarcophagidae e Calliphoridae.

PALOSCHI et al. (1984) mencionaram a Musca domestica e a Fannia sp como vetores biológicos de $\boldsymbol{D}$. hominis no planalto catarinense. RIBEIRO et al. (1985) no Rio Grande do Sul (RS), registraram a Fannia sp., Stomoxys calcitrans, $M$. domestica, Phaenicia sp e Tabanus sp. como vetores biológicos da $\boldsymbol{D}$. hominis, sendo que dos 98 vetores capturados, 93 pertenciam ao gênero Fannia, um ao gênero Phaenicia, duas $M$. domestica, um Tabanus sp. e uma $S$. calcitrans. OLIVEIRA (1986) em São Carlos, Estado de São Paulo, empregando uma armadilha que utilizava um bezerro como isca viva, capturou 59 vetores biológicos da $\boldsymbol{D}$. hominis, dos quais 51 foram identificadas como $M$. domestica, seis do gênero Fannia e duas $\boldsymbol{S}$. calcitrans, todos eles capturados na proximidade de um estábulo. MAIA \& GOMES (1988) na região de Uberaba, Estado de Minas Gerais (MG), capturaram 14 vetores biológicos de $\boldsymbol{D}$. hominis sendo sete $\boldsymbol{M}$. domestica, quatro Sarcophagula sp., duas $\boldsymbol{S}$. calcitrans e uma Fannia $\mathrm{sp}$. dos quais cinco espécimes foram capturados na área do curral, sete em campo aberto e dois na mata. SILVA et al. (1989) no município de Itabuna, Estado da Bahia, numa pesquisa sobre transportadores de ovos da D. hominis, capturaram 75 fêmeas de Sarcopromusca pruna durante os dois anos do período experimental. KASAI et al. (1990) estudando a variação sazonal de dípteros em armadilha modificada de Magoom em Santana do Paraíba, Estado de São Paulo, capturaram um vetor biológico da $\boldsymbol{D}$. hominis pertencente ao gênero Fannia sp. em uma das armadilha colocada perto da mata. LEITE $e t$ al. (1994), notificaram pela primeira vez no Brasil, o envolvimento da Haematobia irritans em amostras obtidas no município de Morada Nova, MG. Três espécimes de Fannia punctipennis (BRUM et al., 1995), foram capturadas em armadilhas de WOT no município de Pelotas, RS. O objetivo deste trabalho foi identificar os dípteros que a $\boldsymbol{D}$. hominis utiliza como vetor biológico nas comunidades de insetos da entomofauna da microregião homogênea de Belo Horizonte.

\section{MATERIAIS E MÉTODOS}

A pesquisa foi realizada no período compreendido entre junho de 1995 e maio de 1996 na Fazenda Modelo da Escola de Veterinária da UFMG no município de Pedro Leopoldo, MG, localizado numa altitude de 882 metros com clima temperado chuvoso (mesotérmico) (ANTUNES, 1986). Para a coleta dos insetos utilizaram-se três armadilhas Magoom (ROBERTS, 1965; RODRÍGUEZ, 1987) as quais foram colocadas, uma próxima aos currais $\left(\mathrm{n}^{\circ}\right.$ 1), uma em campo aberto $\left(n^{\circ} 2\right)$ e outra no interior da mata $\left(\mathrm{n}^{\circ} 3\right)$. Estas armadilhas tiveram como iscas vivas bezerros de aproximadamente 4 meses de idade, os quais eram expostos semanalmente por 48 horas. Realizou-se então, a retirada dos insetos da armadilha com a ajuda de um vidro aspirador e uma rede entomológica. Após a coleta, os mesmos eram transportados ao laboratório. A identificação até o nível de família foi realizada com base em BORROR \& DELONG (1969), e a nível de gênero ou espécie segundo JAMES (1947), GREENBERG (1971), PAMPLONA \& COURI (1989). Os muscídeos vetores biológicos foram identificados pela presença de ovos da $D$. hominis no abdome. Os resultados são mostrados em tabelas que indicam os locais de captura, espécimes coletados e o total de captura com suas respectivas freqüências absolutas e relativas. As famílias foram listadas iniciando por aquelas pertencentes à sub-ordem Nematocera, seguida da Brachycera e Cyclorrhapha

\section{RESULTADOS E DISCUSSÃO}

Observa-se na Tabela 1 as dez ordens de insetos capturadas durante o período experimental. O maior número de artropodes estavam relacionados com a ordem Diptera com $95,73 \%$, e o menor com a ordem Orthoptera com $0,02 \%$ do total de insetos capturados. Esta tendência da armadilha em capturar maior quantidade de membros da ordem Díptera provavelmente deveu-se a dois fatos. O primeiro foi que a ordem Díptera tem sido considerada como uma das mais numerosas da classe Insecta com 85.000 espécie (NEVES, 1989) e a outra, ao tipo de armadilha empregada. Os tipos de armadilhas para captura de insetos têm sido variados, e a sua escolha depende principalmente dos grupamentos de insetos a pesquisar. A armadilha Magoom, pelo tipo de isca utilizada, atrai principalmente insetos zoófilos, sendo que a maioria deles relaciona se à ordem Díptera. Se outro tipo de armadilha como a "pitfall" (RODRÍGUES \& FLECHTMANN, 1995) fosse usada na mesma área de 


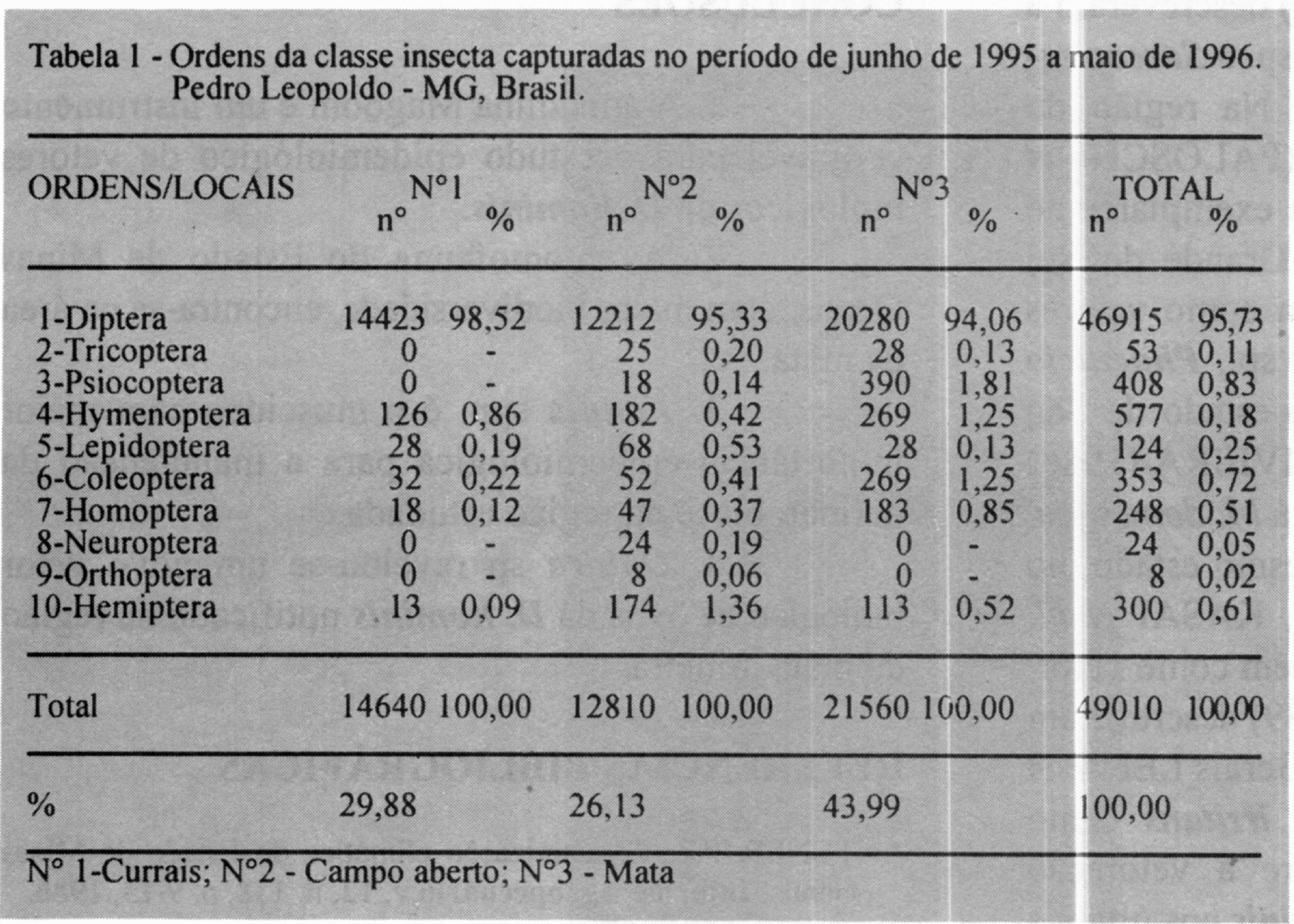

Dos três locais utilizados para coleta o interior da mata (local $n^{0} 3$ ) foi o que teve o maior percentual de insetos capturados $43,23 \%$ do total de dípteros colhidos no período experimental. Estes resultados foram semelhantes aos de DIAS (1983) que obteve uma captura de $52,69 \%$ para a região de Belo Horizonte e com os de PALOSCHI, (1984) que capturou $43,44 \%$ de insetos no planalto catarinense.

Os vetores biológicos da $\boldsymbol{D}$. hominis encontrados neste experimento foram Fannia spp, $M$. domestica, $S$. calcitrans, $S$. nudiseta, Ophira sp. (Tabela 3). No mesmo Estado, na região de

pesquisa, a ordem coleóptera seria provavelmente a que apresentaria maior número de exemplares capturados, devido à especificidade desta para com esta ordem. Existem ainda armadilhas específicas para capturar exemplares de insetos de determinada família como é o caso da armadilha Piramidal (LEPRINCE et al. 1994) para espécies da família Tabanidae ou a armadilha de Vavoua iscada com octenol (MIHOK, et al., 1995) para exemplares do gênero Stomoxys. Neste trabalho, a armadilha de Magoom foi a que ofereceu uma ampla diversidade ao capturar um maior número de famílias das diferentes sub-ordens da ordem díptera.

Dos 49.010 insetos capturados, a Tabela 2 mostra que 46.915 espécimes pertenciam à ordem Díptera, estando distribuídos em 28 famílias, das quais, as famílias Sepsidae, Syrphidae e Culicidae foram as mais numerosas, somando acima de $60 \%$ do total dos dípteros capturados. Apesar destas famílias serem encontradas em maior número não se observou vetores de D. hominis. Contrariamente, as famílias Muscidae e Fannidae, com pequenas quantidades de espécimes coletados $(6,02 \%$ e $4,68 \%$ respectivamente do total), tiveram uma significativa quantidade de espécimes capturadas, ou seja $66,67 \%$ para a família Fannidae e 33,33\% para a família Muscidae do total de amostras positivas para ovos de D. hominis.

\begin{tabular}{|c|c|c|c|c|c|}
\hline FAMÍLIAS/LOCAIS & $\mathrm{N}^{\circ} 1$ & $N^{\circ} 2$ & $N^{\circ} 3$ & TOTAIS & $\%$ \\
\hline \multirow{2}{*}{$\begin{array}{l}\text { 1-Culicidae } \\
\text { 2-Tanvderidae }\end{array}$} & 337 & 4240 & 3701 & 8278 & 1764 \\
\hline & 0 & 147 & 1368 & 1515 & 3.23 \\
\hline 3-Psychodidae & 773 & 501 & 2817 & 4091 & 8,72 \\
\hline 4-Mycetophilidae & 12 & 320 & 333 & 665 & 1,42 \\
\hline 5-Tipulidae & 320 & 370 & 3297 & 3987 & 8.50 \\
\hline 6-Blephariceridae & 0 & 18 & 0 & 18 & 0,04 \\
\hline 7-Chironomidae & 0 & 21 & 22 & 43 & 0,09 \\
\hline 8-Tabanidae & 2 & 12 & 19 & 33 & 0,07 \\
\hline 9-Stratiomyidae & 14 & 28 & 16 & 58 & 0,12 \\
\hline 10-Dolichopodidae & 0 & 45 & 369 & 414 & 0,88 \\
\hline 11-Asilidae & 0 & 8 & 0 & 8 & 0,02 \\
\hline 12-Rhagionidae & 0 & 0 & 49 & 49 & 0,10 \\
\hline 13-Syrphidae & 4661 & 872 & 3162 & 8695 & 18,53 \\
\hline 14-Phoridae & 0 & 29 & 80 & 109 & 0,23 \\
\hline 15-Conopidae & 0 & 0 & 8 & 8 & 0,02 \\
\hline 16-Sepsidae & 4678 & 3688 & 3199 & 11565 & 24,65 \\
\hline 17-Drosophilidae & 0 & 43 & 8 & 51 & 0,11 \\
\hline 18-Chloropidae & 7 & 40 & 0 & 47 & 0,10 \\
\hline 19-Tephritidae & 0 & 35 & 0 & 35 & 0,07 \\
\hline 20-Micropezidae & 0 & 0 & 10 & 10 & 0,02 \\
\hline 21-0. Acaliptrados & 294 & 121 & 441 & 856 & 1,82 \\
\hline 22-Fannidae & 988 & 548 & 662 & 2198 & 4,69 \\
\hline 23-Muscidae & 1742 & 825 & 255 & 2822 & 6,02 \\
\hline 24-Calliphoridae & 234 & 155 & 256 & 645 & 1,37 \\
\hline \multirow{2}{*}{$\begin{array}{l}\text { 25-Sarcophagidae } \\
\text { 26-Tachinidae }\end{array}$} & 169 & 132 & 148 & 449 & 0,96 \\
\hline & 157 & 9 & 8 & 174 & 0,37 \\
\hline $\begin{array}{l}\text { 26-Tachinidae } \\
\text { 27-Anthomyidae }\end{array}$ & 35 & 2 & 52 & 89 & 0,19 \\
\hline 28-Hippoboscidae & 0 & 3 & 0 & 3 & 0.01 \\
\hline $\begin{array}{l}\text { Totais } \\
\%\end{array}$ & $\begin{array}{r}14423 \\
30,74\end{array}$ & $\begin{array}{r}12212 \\
26,03\end{array}$ & $\begin{array}{r}20280 \\
43,23\end{array}$ & $\begin{array}{l}46915 \\
100,00\end{array}$ & 100,00 \\
\hline $\begin{array}{l}\text { De } 1 \text { a } 7 \text { - nematoce } \\
\text { De } 8 \text { a } 12 \text { - brachyc } \\
\text { De } 13 \text { a } 28 \text { - cyxlor }\end{array}$ & & & & & \\
\hline
\end{tabular}


Uberlândia (MAIA \& GOMES, 1988) descreveram a M. domestica, S. calcitrans, Fannia sp. e Sarcophagula sp. como vetores biológicos. Na região do planalto do Estado de Santa Catarina (PALOSCHI, et al. 1984) reportaram $M$. domestica e exemplares do gênero Fannia. No estado de Rio Grande do Sul (RIBEIRO et al. 1985) apresentaram como vetores biológicos, a M. domestica, Fannia sp., Phaenicia sp., S. calcitrans e Tabanus sp. No estado de São Paulo, município de São Carlos, OLIVEIRA (1986) descreveu como os vetores biológicos á M. domestica, Fannia spp. e $\boldsymbol{S}$. calcitrans, e no mesmo estado, no município de Santana do Paranaiba, KASAI et al. (1990) encontraram a Fannia sp também como vetor. No estado da Bahia, SILVA et al. (1989) descreveram a Sarcopromusca pruna. Em Minas Gerais LEITE et al. (1994) notificaram Haematobia irritans como vetor biológico. As pesquisas sobre a vetoração biológica de muscídeos para a $\boldsymbol{D}$. hominis nos últimos 15 anos no Brasil, indicam o gênero Fannia como principal gênero de importância epidemiológica para a dermatobiose no país, uma vez que $56,61 \%$ dos vetores coletados pertencem a este agrupamento, seguida pela $\boldsymbol{M}$. domestica que representa $19,35 \%$ do total das vetorações registradas (PALOSCHI et al., 1984; RIBEIRO et al., 1985; OLOVEIRA, 1986; MAIA \& GOMES 1988; SILVA et al., 1989). GUIMARÃES et al. (1983) apresenta a última lista atualizada dos vetores de ovos de $\boldsymbol{D}$. hominis, nesta lista não consta o registro o gênero $\boldsymbol{O}$ phyra como carregadora de ovos de D. hominis, dentro dos espécimes pertencentes à família Muscidae. Neste trabalho incorpora-se o gênero Ophira sp como novo vetor biológico de $\boldsymbol{D}$. hominis

\begin{tabular}{|c|c|c|c|c|c|c|c|c|}
\hline \multirow[t]{2}{*}{ VETORESES/LOCAIS } & \multicolumn{2}{|c|}{$\mathrm{N}^{\circ} 1$} & \multicolumn{2}{|c|}{$N^{\circ} 2$} & \multicolumn{2}{|c|}{$N^{\circ} 3$} & \multicolumn{2}{|c|}{ TOTAL } \\
\hline & $n^{\circ}$ & $\%$ & $\mathrm{n}^{\circ}$ & $\%$ & $\mathrm{n}^{\circ}$ & $\%$ & $n^{\circ}$ & $\%$ \\
\hline $\begin{array}{l}\text { 1-Fannia spp. } \\
\text { 2-Sarcopromusca pruna } \\
\text { 3- Musca domestica } \\
\text { 4- Ophira sp. } \\
\text { 5- Stomoxys Calcitrans }\end{array}$ & $\begin{array}{r}10 \\
9 \\
21 \\
8 \\
12\end{array}$ & $\begin{array}{l}17 \\
15 \\
35 \\
13 \\
20\end{array}$ & $\begin{array}{l}9 \\
0 \\
0 \\
0 \\
0\end{array}$ & $\begin{array}{c}100 \\
- \\
- \\
-\end{array}$ & $\begin{array}{l}81 \\
0 \\
0 \\
0 \\
0\end{array}$ & $\begin{array}{c}100 \\
- \\
- \\
-\end{array}$ & $\begin{array}{r}100 \\
9 \\
21 \\
8 \\
12\end{array}$ & $\begin{array}{r}66,67 \\
6,00 \\
14,00 \\
5,33 \\
8,00\end{array}$ \\
\hline $\begin{array}{l}\text { Totais } \\
\%\end{array}$ & $\begin{array}{l}60 \\
40\end{array}$ & 100 & $\begin{array}{l}9 \\
8 .\end{array}$ & 100 & $\begin{array}{l}81 \\
54\end{array}$ & 100 & $\begin{array}{c}150 \\
100,0\end{array}$ & $\begin{array}{l}100,00 \\
0\end{array}$ \\
\hline
\end{tabular}

$\mathrm{N}^{\circ} 1$ - Currais; $\mathrm{N}^{\circ} 2$ - Campo aberto; $\mathrm{N}^{\circ} 3$ - Mata.

\section{CONCLUSÕES}

1. A armadilha Magoom e um instrumento confiável para o estudo epidemiológico de vetores biológicos da $\boldsymbol{D}$. hominis.

2. A entomofauna do Estado de Minas Gerais, com maior biodiversidade, encontra-se na área da mata.

3. Fannia spp. é o muscideo com maior importância epidemiológica para a manutenção da dermatobiose na região estudada.

4. Ophira sp revelou-se um novo vetor biológico de ovos da $\boldsymbol{D}$. hominis notificado na região da mata mineira.

\section{REFERÊNCIAS BIBLIOGRÁFICAS}

ANTUNES, F.Z. Caracterização climática do Estado de Minas Gerais. Informe Agropecuário,v. 12, n. 138, p. 9-13, 1986.

ARTIGAS, P. T., SERRA, R.G. Portadores de ovos de Dermatobia hominis (1. Jr., 781). Atualização da lista de foréticos, com enumeração de novos agentes do "Berne". Ciência \& Cultura, v. 17, n. 1, p. 21-29, 1965.

BORROR, D.J., DELONG, D.M. An introduction to the study of insects. 3 ed. New York: Rinehart and Winston, 1969, 400 p.

BRUM, J.G.W., GENTILINI, F., PINTO, L.S., et al. Fannia punctipenis (Díptera:Muscidae), vetor de ovos da Dermatobia hominis (Oestridae: cuterebrinae) em Pelotas, RS. In: SEMINÁRIO BRASILEIRO DE PARASITOLOGIA VETERINÁRIA, 1995. Campo Grande, MS. Anais... Campo Grande, Colegio Brasileiro de Parasitologia Veterinária, 1995, 175 p. p. 92.

DIAS, E.S. Levantamento taxonômico e sinantrópico da fauna de Sarcophagidae (Díptera) em Belo Horizonte, Minas Gerais.Belo Horizonte:Universidade Federal de Minas Gerais, 1982. 91 p. Dissertação (Mestrado em Parasitologia)- Curso de Pós-graduação em Parasitologia, Universidade Federal de Minas Gerais, 1982.

DUNN, L.H. The tick as a possible agent in the collocation of the eggs of Dermatobia hominis (Linn.). Journal of Parasitology, v. 4, p. 154-158, 1918.

GREENBERG, B. Flies and diseases; ecology, classifications and biotic associations. Princenton Univ. Press, Princeton. $1971.856 \mathrm{p}$.

GUIMARÃES, J.H., PAPAVERO, N. A tentative annoted bibliography of $\boldsymbol{D}$. hominis (Linnaeus, Jr., 1781) (Díptera:Cuterebridae). Arquivos de Zoologia, v. 14, n. 4, p. 223- 294, 1966.

GUIMARÃES, J.H., PAPAVERO, N., PRADO. A.P. do As miíases da região neotropical. Revista Brasileira de Zoologia, v. 1, n. 4 , p. 239-416, 1983.

JAMES, M.T. The flies that cause myasis in man. USDA. Washington. D.C. 1947. $175 \mathrm{p}$. 
KASAI, N., SCHUMAKER, T.T.S., DELL'PORTO, A. et al. Variação sazonal de dípteros capturados em armadilhas de Magoom modificada, em Santana de Parnaíba, Estado de São Paulo. Revista Brasileira de Entomologia, v. 34, p. 369-380, 1990.

LEITE, R.C., RODRÍGUEZ,B.Z., OLIVERIRA, P.R. et al. Haematobia irritans (L.) (Diptera:Muscidae) mais um transmissor biologico de Dermatobia hominis (L.). Brasil,1993. In: CONGRESSO BRASILEIRO DE MEDICINA VETERÍNÁRIA. 23. 1994, Pernanbuco. Anais... Pernanbuco. SBMV, 1994; 195 p., p. 92.

LEPRINCE, D.J., HRIBAR, L.J., FOIL,L.D. Responses of horse flies (Diptera:Tabanidae) to Jersey bullocks and canopy traps baited with ammonia, octenol, and carbon dioxide. Journal of Medical Entomology. v. 31, p. 729-731, 1994.

MAIA, A.A.M., GOMES, A.G. Vetores de Dermatobia hominis, Linnaeus Jr., 1781 (Díptera:Cuterebridae) na região de Uberaba, Minas Gerais. Revista da Faculdade Medicina Veterinaria e Zootecnia, v. 25, n. 1, p. 47-51, 1988.

MIHOK, S., E.K. KANG'ETHE., G. K. KAMAU. Trials of traps and attractants for Stomoxys spp. (Diptera:Muscidae). Journal of Medical Entomology, v. 32, n. 3, p. 283-289, 1995.

NEEL, W. W., URBINA, O., VIALE, E. et al. Ciclo biologico del tórsalo (Dermatobia hominis L.J.) en Turrialba, Costa Rica. Turrialba, v. 5, n. 3, p. 91-104, 1955.

NEIVA, A., GOMES, J.F. Biologia da mosca do berne (Dermatobia hominis) observada em todas sus phases. Annaes Paulista de Medicina e Cirugia, v. 8, n. 9, p. 197- 209, 1917.

NEVES,D.P. Entomologia médica: Comportamento, captura, montagem.Belo Horizonte:Coopemed, $1989.112 \mathrm{p}$

OLIVEIRA, G.P. Distribuição sazonal de dípteros muscóides sinantrópicos, Simbovinos e foréticos de Dermatobia hominis L. Jr., em São Carlos, Estado de São Paulo. I Estábulo. Arquivo de Biologia e Tecnologia, v. 29, n. 2, p. 311-325, 1968

PALOSCHI, C.G., RAMOS, C.I., SOUZA, A.P. et al. Vetores de ovos da Dermatobia homini (Diptera: Cuterebridae) no planalto catarinense. In: CONGRESSO BRASILEIRO DE MEDICINA VETERINÁRIA, 1984. Belem, PA. Anais... Belém, Colégio Brasileiro de Medicina Veterinária, 1984, 109 p. 71.

PAMPLONA, D., COURI, S.M. Revisão das espécies neotropicais de Ophyra Robineau-Desvoidy, 1830 (Diptera, Muscidae, Azelinae). Memórias do Instituto Oswaldo Cruz, v. 84, s. IV, p. 419-429, 1989.

RIBEIRO, P.B., OLIVEIRA, C.M.B., COSTA, P.R.P, et al. Foréticos da Dermatobia hominis (1.Jr., 1781) (Díptera: Cuterebridae) no Rio Grande do Sul, Brasil. Arquivos Brasileiro de Medicina Veterinaria e Zootecnia, v. 37, n. 5, p. 507 509,1985 .

ROBERTS, R.H. A steer-baited trap for sampling insects affecting cattle. Mosquito News. v. 25, p. 281-285, 1965.

RODRÍGUES, S.R., FLECHTNMANN, C.A.H. Levantamento de besouros coprófago (Coleoptera: Scarabaeidae) em Pereira Barretos, SP. In: CONGRESSO DE ENTOMOLOGIA, 1995. Caxambu, MG. Anais... Caxambu, Sociedade Brasileira de Entomologia, 1995,635 p. p.580.

RODRÍGUEZ, B..Z. Ocorrência e distribuição de larvas de Dermatobia hominis (L. Jr., 1781) em bovinos e flutuação de Dípteros sinantrópicos rurais. Pelotas: Universidade Federal de Pelotas, 1987. 70 p. Dissertação (Mestrado en Sanidade Animal) - Curso de Pós-graduação em Sanidade Animal, Universidade Federal de Pelotas, 1987.

SILVA, A.A., SMITH, D.H., BARBOSA, S.A.J.S. Sarcopromusca pruna (Diptera: Muscidae) as an egg transport host of Dermatobia hominis (Diptera: Cuterebridae) in the cacau region of Bahia, Brasil. Memorias do Instituto Oswaldo Cruz, v. 84, s. IV, p. 491-497, 1989.

Ciência Rural, v. 27, n. 4, 1997. 